\title{
The Research of Theoretical and Practical Teaching of Synthetic Crystal Based on the Dual Employment Patterns
}

\author{
Wei Zheng ${ }^{1,}$, Xiulan He, Yexia Fan ${ }^{1}$ \\ ${ }^{1}$ School of Materials Science and Engineering, Harbin University of Science and Technology, \\ Harbin, 150040, P. R. China \\ aemail: zhengwei1972@sina.com
}

Keywords: the profession of inorganic nonmetallic materials; synthetic crystal; teaching reform; dual employment pattern

\begin{abstract}
The synthetic crystal plays a key role in technology development and daily life. Recently, the employment of inorganic nonmetallic material graduates transform gradually from single direction to diversification. And the teaching method of the synthetic crystal course is being required to adapt this situation urgently. The necessity of the course construction has been analyzed. According to the teaching objectives and existing problems, specific reform measures have been proposed including textbook construction, experimental teaching reform, educational method innovation and evaluating mode regulation. The approaches worthy of reference are provided to stimulate learning interest, master the basic knowledge and technique flexibly for students to meet the employment requirement.
\end{abstract}

\section{Introduction}

Synthetic crystal, as a significant branch of inorganic nonmetallic materials, is a crystal synthesized by manual method. Crystallography is one of the most foundational courses in inorganic nonmetallic materials field, meanwhile the synthetic crystal course is also necessary theoretical branch of crystallography. So it is important to set up the courses in the higher education of inorganic nonmetallic profession. But there are some problems existed in synthetic crystal: the short research history, the deep professional skills, the incomplete teaching system and so forth. And how to improve the teaching system and quality, how to provide enough knowledge content in limit curriculum time and how to stimulate the learning interest of students to grasp more useful knowledge effectively are becoming the practical issues urgently to be solved. Thus the reformation and innovate of the synthetic crystal courses must be put into practice under this situation.

\section{Curriculum Objectives and Recent Situation}

\section{The Curriculum Objectives}

Synthetic crystal course is not only the basis of the inorganic nonmetallic profession, but also the other majors of the Materials Science and Engineering ${ }^{[1]}$. So we target the main curriculum projects, according to the profession designing and the importance of the course, as follows:

- Mastering the basic theoretical knowledge and principle of synthetic crystal.

- Combining the teaching and practice and cultivating the practical abilities.

- Developing the interest of students in scientific research and the capabilities of solving problems, and understanding academic foreland.

-Expanding knowledge domain and achieving the interdisciplinary research.

It not only makes students accumulate the basic knowledge in synthetic crystal, but also can be applied to practice. Meanwhile, developing interest is the best way to research professional knowledge deeply and build the internal relation between disciplines to expand horizons ${ }^{[2]}$.

\section{Recent Situation of Curriculum}

The course, Synthetic Crystal, has been established as the grundstudium specific to the 
profession of inorganic nonmetallic materials in our academy recently. But the theoretical knowledge in the textbook which is the book used to scientific research is too abstruse to understand. And only the experiment "Synthesis and Properties of KDP Crystals" has been acted as relevant experimental curriculum. So three issues existed in the curriculum mainly:

- Lack of vary course installation and specific theoretical textbooks

Due to the deep textbook, the limit understanding for students and lack of providing related cutting-edge research progress, the disconnection between theory and practice become more serious. Besides, the students just want to pass the exams and get credit, there are no more interests in acquiring knowledge to meet employment demand.

-Lack of practice teaching, conventional laboratory instruments and equipment supply, it is difficult to achieve combination of theory and practice

The basic experimental classes in our experimental teaching are in a repeating pattern, the content is not only less involved to further deepen the understanding of theoretical knowledge, but also lack of new points and technology. Coupled with hysteretic laboratory equipment and the laboratory instruments which cannot be replenished in time, all of these reasons bring difficulties to the basic experimental design. And the experimental methods, laboratory supplies and even experimental procedures are provided in detail in the textbook before the experiments in the usual experimental courses ${ }^{[3]}$. So it is a question for the students to improve learning initiative, creativity and the ability to work independently. Due to inadequate investment in teaching, theoretical teaching are often taken seriously, but practical teaching despised, such as curriculum design, professional training, productive practice and graduation design ${ }^{[4]}$.

- Teaching methods and mode immobilization, students are not enthusiastic

At this stage, the synthetic crystal classroom instruction is still using traditional teaching methods, flat one-way transfer, that knowledge is taught by teachers, notes are written on the blackboard and traditional teaching media are applied. And teaching content is taught to students, intentionally or unintentionally, in the teaching process to strengthen the domination and control status of teachers, and students as the passive receivers ${ }^{[5]}$. The result of this situation only lead to seriously reduce the enthusiasm of the students and increase negative emotion to cope with exams, not to mention the interest in-depth knowledge and scientific knowledge of the course. Finally the teaching effect cannot be achieved naturally.

Based on the above problems of synthetic crystal courses, raising reasonable teaching methods, practical and feasible teaching reform become a urgent project.

\section{Reforming Measures of the Curriculum}

We are exploring comprehensively on the courses construction, according to the strong professional and technical, theoretical knowledge of the abstract and complex and multidisciplinary, and other features of the synthetic crystal courses, involving the construction of textbooks, practical teaching reform, teaching methods innovation and adjustment in the form of assessment and other links.

\section{Proceeding in Textbook Construction and Curriculum Restrucuring}

For the current professional theoretical knowledge mismatching the textbooks, the task group will re-design knowledge structure, write teaching materials suitable for undergraduate and graduate students. It involves types of synthetic crystal (such as laser crystal, semiconductor crystals, scintillation crystal and so on), the synthetic growth mechanism and applications basic expertise, the content related to the advanced cutting-edge knowledge added so that students can learn about the latest developments and practical applications of the synthetic crystal. And the timely preparation of bilingual materials and jargon marked on the basis of the English summary make students learn expertise with knowing English terminology.

It is necessary to establish in addition relevant curriculums such as "Modern Crystal", "Crystal Topography", "Crystal Technology" etc. on the basis of "Synthetic Crystal", in order to broaden the knowledge. Besides, frontier newspapers, like "Journal of Synthetic Crystals", need to be provided 
for students to read. Reasonable curriculum arrangement under the professional direction need to be proceeded to break the fixed limitations of the situation and to develop the flexibility and diversity.

\section{Optimizing of the Experimental System and Emphasizing on Practice}

Our academy has the synthetic crystal research laboratory with crystal growth and processing equipments: single crystal furnaces, crystal orientation analyzer, the inner circle cutting machine, two axis grinding machines. But the visible experimental teaching conditions are still inadequate, so we must purchase conventional experimental teaching instruments to facilitate the teaching and design of experiments. Laboratory equipments are managed specifically by professional teachers, but at a certain time open to students to meet the needs of different people.

Practice teaching is a key link of the students in directly contacting with the actual work and exercising capacity, and it has a very important significance for students to recognize the social and change thought. Thus the research team carefully plans artificial crystal practice teaching content. Practice bases of photoelectric field, such as communication, are added in production practice stage of inorganic non-metallic materials profession not only to enable students to understand the basic situation of the synthetic crystal in the field of production, but also to make students ideologically transfer from the experimental to the engineering field. Closing to the actual crystal materials engineering, we will arrange diversiform and comprehensive subjects, and allow students hands-on operation to deepen and consolidate students' understanding of the professional courses. Graduation design is no longer limited to the design of the traditional inorganic non-metallic materials, it is necessary to take full advantage of the artificial crystal laboratory resources to design topics which can reflect the cutting-edge work in the photoelectron field, such as " the application of photonic crystal technology in optical fiber communication devices ", that allows students to contact the research frontier and cultivate them research quality and capacity.

\section{Innovating of the Teaching Mode and Methods}

As mentioned before, the traditional teaching mode cannot meet the needs of modern culture, so that teachers should be actively trying to adopt more modern teaching methods, such as: discussion, questions or observing teaching, multimedia teaching and interactive teaching etc. to change the mode of "one-man show" [6]. In this way students can continue to ask questions or express their opinions in the classroom. The teachers need to mobilize the thinking of students, and students may also have the article or panel discussion on some deeper theoretical issues, that questions asked by the teachers in advance, students require to discuss by previewing textbooks, reference books or professional journals, and then to write papers, proceed group discussion in the class and speak by the representative.

Because of the abstract crystal structure and morphology, it is difficult for students to imagine, so in addition to the general multimedia plane teaching, our research team intends to produce three-dimensional format courseware that can be downloaded from the school website. For example, three-dimensional GIF format images can be constantly rotated slowly and the relationship of the spatial distribution between crystal morphology from any angles and spatial point group. It can greatly improve the effectiveness of teaching. Also the teachers show video or flash courseware content, such as the production process records, teaching videos at home or abroad and the latest reported research results of crystal, all contribute to develop students' interest. And multimedia teaching on the practical lessons can also be applied to the artificial crystal courses. For example, teachers demonstrate crystal optical phenomena for the students by connecting the polarizing microscope and computer projector to achieve microscopic interactive teaching that teachers and students can communicate on the microscope at any time seeing the phenomenon through the images on the computer. Experimental multimedia teaching will improve teaching efficiency and quality.

\section{Adjusting the Evaluation Form and Stimulating Learning Enthusiasm}

Course examinations in university level must not just look at the mark of the final papers, this can only lead to the situation of dealing with the exam. We should improve learning enthusiasm subjectively in the reform and innovation of teaching methods and also should improve the 
assessment approach to change the attitude of students objectively. Therefore, in theoretical courses, teachers should be based on usual attendance, classroom speaking, discussions, expressing opinions and writing papers to allocate the appropriate proportion of marks (70\%) with the results of the written test (30\%) as the final examination mark. This can stimulate the enthusiasm of active learning. In experimental courses, teachers should be based on previewing reports, lab reports, classroom discussion, operations and solving the problems successfully in the process of the experiment as usual experimental mark. And the final exam involves a basic experimental skills assessment, if including self-designed experiments, awarded marks will be given. Besides, the performance in practice teaching will be also included in the total credits of college to enable students to take seriously and nurture the overall quality for future employment.

\section{Conclusion}

Based on the current employment situation of inorganic non-metallic materials profession, the demand for professionals is gradually expanding in synthetic crystal direction. While, our pedagogical purpose is that cultivate high-quality professional talents with initiative and creativity to promote the career development of students. Teaching content and methods presented in this paper is based on the employment to be adjusted continually, in order to form a "dynamic" curriculum mode and teaching methods. Although our course construction has just begun, in some ways it has achieved initial achievement. We firmly believe that the teaching quality and effectiveness of the synthetic crystal curriculum will be further enhanced with the gradual deepening of the course construction.

\section{Acknowledgement}

This work is financially supported by the " Twelfth Five-year" plan of the Higher Education Research, "The Teaching and Experimental Research of Synthetic Crystal upon the Dual Employment Directions of Inorganic Nonmetallic Materials “, under the Institute of Higher Education in Heilongjiang Province. (Project No: HGJXHC110546)

\section{References}

[1] Ying Zhang. The reform and innovation of crystallography under the big material background (Chinese) [J] . Education Science \& Culture, 2011, 10(28), 71-72.

[2] Cuihong Hou, Jie Zhang, Weihang Chen, Jianwei Tang, Baozeng Ren, Yazhen Wan, Yanliang Luo. Reform on the curriculum system and teaching content of Chemical Engineering and Technology (Chinese) [J] .Higher Education of Science, 2012, 4 : 115-118.

[3] Guangpei Tian, Qian Xu. Summary of university experimental teaching reform (Chinese) [J] . China Education Research Analects, 2007(00): 151-153.

[4] Jugong Zheng, Quanshui Chen, Xiaodong Liu, Yang Ting, Yunhai Liu, Guangyuan Ren. On the construction of training model and curriculum system of applied and innovative talents majoring in materials (Chinese) [J] . Higher Education of Science, 2011, 6: 143-147.

[5] Ming Chen, Zhizheng Lei, Caiyan Wang. A teaching model which combines teaching and research (Chinese) [J] . Journal of Guilin University of Electronic Technology, 2008, 28(2): 163-166.

[6] Yingxie Jin. Discuss the reform of the teaching mode (Chinese) [J] . China Education Research Analects, 2006(00): 105-106. 